\title{
NONLINEAR ELLIPTIC FUNCTIONAL EQUATIONS IN NONREFLEXIVE BANACH SPACES
}

\author{
BY FELIX E. BROWDER ${ }^{1}$ \\ Communicated October 7, 1965
}

Consider a strongly elliptic quasilinear system of partial differential operators on an open subset $\Omega$ of $R^{n}$ in generalized divergence form:

$$
A(u)=\sum_{|\alpha| \leq m} D^{\alpha} A_{\alpha}\left(x, u, \cdots, D^{m} u\right) .
$$

If the coefficient functions $A_{\alpha}$ have at most polynomial growth in $u$ and its derivatives, we have shown in [1] how existence theorems for solutions of variational boundary value problems for the equation $A(u)=f$ may be obtained from monotonicity assumptions on the nonlinear Dirichlet form of $A$, with extensions to weaker ellipticity assumptions in [3], [4], [9], and [15]. These results extend earlier theorems of Višik [18] obtained by other arguments, but (despite an apparent remark to the contrary in [15]) do not extend automatically to the case of rapidly increasing coefficients treated by Višik in [19]. The crucial point for this more general case is that the Banach spaces in which the problems are appropriately formulated are derived from nonreflexive Orlicz spaces [14] and are themselves nonreflexive and nonseparable. On the other hand, the basic treatments of monotone and semimonotone operator equations have been carried out in reflexive Banach spaces (and in [15], it is even essential to consider only separable spaces).

It is our object in the present note to outline a treatment of these more general problems in nonreflexive spaces, using the remark already made by the writer in the final section of [4] on the useful properties of the weak* topology on a conjugate space. We formulate this treatment in a very general context of functional rather than simply differential equations, and apply to the resulting operator equations a new theorem on perturbation of semimonotone operators (Theorem 2 below) which is particularly efficacious in this general context. This result is extended and applied in more detail in [11] to include all the elliptic results of [1], [3], [4], [9], [15], [18], and [19], and a corresponding extension to unbounded operators and to monotone operators on convex sets gives a significant extension of 3552 .

${ }^{1}$ The preparation of this paper was partially supported by N.S.F. Grant GP. 
the results on nonlinear initial value problems developed in [2], [5], [7], and [9], (as well as of recent results for monotone operators on convex sets of the writer [10] and Hartman and Stampacchia [13]).

1. Suppose that for each multi-index $\alpha$ with $|\alpha| \leqq m$, we are given a Banach space $X_{\alpha}$ of distributions on $\Omega$ with its dual space $X_{\alpha}^{*}$ also a space of distributions and $C_{c}^{\infty}(\Omega) \subset X_{\alpha}^{*}$. We set $X=\prod_{\alpha} X_{\alpha}, X^{*}$ $=\prod_{\alpha} X_{\alpha}^{*}$. Corresponding to this family of spaces, we have the generalization of the Sobolev spaces given by

$$
W_{X^{*}}=\left\{u \mid u \in D^{\prime}(\Omega), D^{\alpha} u \in X_{\alpha}^{*} \text { for }|\alpha| \leqq m\right\} .
$$

Let $\zeta$ be the mapping of $W_{X^{*}}$ into $X^{*}$ given by $\zeta(u)=\left\{D^{\alpha} u|| \alpha \mid \leqq m\right\}$. We norm $W_{X^{*}}$ so that $\zeta$ is an isometry. Then $\zeta$ identifies $W_{X^{*}}$ with a weak* closed subspace of $X^{*}$.

The data of a nonlinear elliptic functional equation are the following: We are given a subspace $V_{0}$ of $W_{X^{*}}$ such that $\zeta\left(V_{0}\right)$ is weak* closed in $X^{*}$, an element $f$ of $X$, and a continuous mapping $A_{\alpha}$ for each $\alpha$ of $X^{*}$ into $X_{\alpha}$. We define the nonlinear Dirichlet form $a(u, v)$ for each $u$ and $v$ in $V_{0}$ by

$$
a(u, v)=(A(\zeta(u)), \zeta(v))
$$

where $A$ is the product map of $X^{*}$ into $X$ with components $A_{\alpha}$ and $(\cdot, \cdot)$ denotes the pairing between $X$ and $X^{*}$. The corresponding boundary problem asks for an element $u$ in $V_{0}$ such that for all $v$ in $V_{0}$, we have:

$$
a(u, v)=(f, \zeta(v)) .
$$

THEOREM 1. Suppose that the Dirichlet form $a(u, v)$ satisfies the two conditions:

(1) For all $u$, $v$ of $V_{0}$

$$
a(u, u-v)-a(v, u-v) \geqq 0 .
$$

(2) $A s\|u\| \rightarrow+\infty$,

$$
a(u, u) /\|u\| \rightarrow+\infty .
$$

Then the above boundary value problem has a solution for every $f$ in $X$.

Proof of Theorem 1. Let $V_{0}^{0}$ be the polar of $\zeta\left(V_{0}\right)$ in $X$, and let $V$ be the quotient space $X / V_{0}^{0}, \tau$ the natural map of $X$ on $V$. Then $\tau^{*}$ is an isometric map of $V^{*}$ into $X^{*}$, and by the weak* closedness of $\zeta\left(V_{0}\right)$, it follows that $\tau^{*}\left(V^{*}\right)=\zeta\left(V_{0}\right)$. Hence, the mapping $\left(\tau^{*}\right)^{-1} \zeta$ is a well-defined isometric isomorphism of $V_{0}$ on $V^{*}$, and if for $u$ and $v$ in $V_{0}$, we set $u^{*}=\left(\tau^{*}\right)^{-1} \zeta(u), v^{*}=\left(\tau^{*}\right)^{-1} \zeta(v)$, we see that 


$$
a(u, v)=(A(\zeta(u)), \zeta(v))=\left(A \tau^{*} u^{*}, \tau^{*} v^{*}\right) .
$$

Hence the condition that $u$ be a solution of the functional equation (3) above is equivalent to the condition that the corresponding $u^{*}$ satisfy:

$$
\left(A \tau^{*} u^{*}, \tau^{*} v^{*}\right)=\left(f, \tau^{*} v^{*}\right)
$$

for all $v^{*}$ in $V^{*}$, i.e.

$$
\left(\tau A \tau^{*}\left(u^{*}\right), v^{*}\right)=\left(\tau(f), v^{*}\right),
$$

or $T\left(u^{*}\right)=\tau A \tau^{*}\left(u^{*}\right)=\tau(f)$, with $T=\tau A \tau^{*}$.

The conditions (1) and (2) above are equivalent to the following conditions for the continuous mapping $T$ of $V^{*}$ into $V$ :

(1)' For each pair $f$ and $g$ of $V^{*}$,

$$
(T f-T g, f-g) \geqq 0 .
$$

$(2)^{\prime}$ As $\|f\| \rightarrow+\infty,(T f, f) /\|f\| \rightarrow+\infty$.

The original problem will have a solution for a given $f$ in $X$ if the corresponding $\tau(f)$ lies in the range of $T$. However, the range of $T$ is all of $V$ by Theorem 5 of [4]. q.e.d.

2. A corresponding translation process for the case where we distinguish between the dependence of the Dirichlet form $a(u, v)$ on the highest and the lower order derivatives of $u$ reduces the corresponding existence result to Theorem 2 below. We preface the statement of the theorem with the following definition: A Banach space $V$ is said to be improvable if there exists a continuous map $M$ of $V^{*}$ into $V$ mapping bounded sets into bounded sets which is monotone (i.e. $(M u-M v, u-v) \geqq 0$ for all $u$ and $v$ ), coercive (i.e. $(M u, u) /\|u\| \rightarrow+\infty$ as $\|u\| \rightarrow+\infty)$ and such that if for a sequence $\left\{u_{k}\right\}$ in $V^{*}$, we have $\left(M u_{k}-M u, u_{k}-u\right) \rightarrow 0$, then $u_{k}$ converges strongly to $u$.

Theorem 2. Let $V$ be an improvable Banach space, $T$ a mapping of $V^{*}$ into $V$ such that $(T u, u) /\|u\| \rightarrow+\infty$ as $\|u\| \rightarrow+\infty$. Suppose that there exist mappings $S$ of $V^{*} \times V^{*}$ into $V$ and $C$ of $V^{*}$ into $V$ for which $T(u)=S(u, u)+C(u)$ for all $u$ in $V^{*}$ and which satisfy the following conditions:

(1) For each fixed $v$ in $V^{*}, S(\cdot, v)$ is a continuous monotone mapping of $V^{*}$ into $V$, i.e. $\left(S\left(u_{1}, v\right)-S(u, v), u-u_{1}\right) \geqq 0$ for all $u$ and $u_{1}$ in $V^{*}$.

(2) For each fixed $u$ in $V^{*}, S(u, \cdot)$ is completely continuous, i.e. continuous from the weak* topology of $V^{*}$ to the strong topology of $V$.

(3) $C$ is compact, i.e. $C$ is continuous from $V^{*}$ to $V$ and maps each bounded subset of $V^{*}$ into a strongly precompact subset of $V$.

(4) $S$ dominates $C$ in the following sense: If $\left\{u_{k}\right\}$ is a sequence in 
$V^{*}$ with $u_{k}$ converging weak* to $u$, and if $\left(S\left(u_{k}, u_{k}\right)-S\left(u, u_{k}\right), u_{k}-u\right)$ $\rightarrow 0$, then $C\left(u_{k}\right)$ converges weakly to $C(u)$.

Then $R(T)$, the range of $T$, is all of $V$.

Corollary to TheOREM 2. The condition (4) may be replaced by the weaker condition:

$(4)^{\prime}$ For every closed ball $B$ about the origin in $V^{*}, T(B)$ is closed in $V$.

REMARK. It can be shown by simple examples that (1), (2), and (3) alone do not imply the conclusion that $R(T)=V$, even when $V$ is a Hilbert space.

Proof of Theorem 2. Since we may subtract a fixed element $w$ of $V$ from $T(u)$ without altering the hypotheses, it suffices to show that $0 \in R(T)$.

We show first that under hypotheses (1), (2), and (3), condition (4) implies $(4)^{\prime}$. In the subsequent argument, only condition $(4)^{\prime}$ will be applied. We use the following fact in this and later proofs (cf. [4]): If $Q$ is a continuous map of $V^{*}$ into $V$ and if for given $u_{0}$ in $V^{*}, w_{0}$ in $V$, and all $u$ in $V^{*}$, we have $\left(Q(u)-w_{0}, u-u_{0}\right) \geqq 0$, then $Q\left(u_{0}\right)=w_{0}$.

PROOF OF $(4)^{\prime}$ FROM (4). Let $\left\{u_{k}\right\}$ be a sequence in the closed ball $B$ such that $T u_{k} \rightarrow w$. (We denote strong convergence by $\rightarrow$ as usual, weak* convergence in $V^{*}$ or weak convergence in $V$ by $\rightarrow$.) Since $B$ is weak* compact, we may assume that $u_{k} \rightarrow u, u \in B$. It suffices to show that $T u=w$. Since $C$ is compact, we may assume also that $C\left(u_{k}\right) \rightarrow w_{1}$. Then $S\left(u_{k}, u_{k}\right)=T\left(u_{k}\right)-C\left(u_{k}\right) \rightarrow w-w_{1}$, and by the complete continuity of $S$ in its second variable, $S\left(u, u_{k}\right) \rightarrow S(u, y)$. Hence

$$
\left(S\left(u_{k}, u_{k}\right)-S\left(u, u_{k}\right), u_{k}-u\right) \rightarrow\left(w-w_{1}-S(u, u), u-u\right)=0 .
$$

Applying condition (4), we have $C\left(u_{k}\right) \rightarrow C(u)$, i.e. $C(u)=w_{1}$. Finally for any $v$ in $V^{*}$, we have for every $k \geqq 1$,

$$
\left(S\left(u_{k}, u_{k}\right)-S\left(v, u_{k}\right), u_{k}-v\right) \geqq 0 .
$$

As $k \rightarrow+\infty, S\left(u_{k}, u_{k}\right) \rightarrow w-C(u), S\left(v, u_{k}\right) \rightarrow S(v, u)$, and $u_{k}-v \rightarrow u$ $-v$. Hence

$$
\left(S\left(u_{k}, u_{k}\right)-S\left(v, u_{k}\right), u_{k}-v\right) \rightarrow(w-C(u)-S(v, u), u-v) \geqq 0 .
$$

Applying our earlier remark with $Q(u)=S(v, u)$, we see that $S(u, u)$ $=w-C(u)$, i.e. $T(u)=w$, and $T(B)$ is closed. q.e.d.

Lemma. For each $\lambda>0$, let $T_{\lambda}=T+\lambda M$, where $M$ is the mapping given in the definition of the improvability of $V$. To prove Theorem 2 , it suffices to show that $0 \in R\left(T_{\lambda}\right)$, for each $\lambda>0$. 
Proof of The Lemma. There exists a function $c(r)$ on $R^{1}$ with $c(r) \rightarrow+\infty$ as $r \rightarrow+\infty$ such that $(T u, u) \geqq c(\|u\|)\|u\|$. For each $\lambda>0$, $\left(T_{\lambda} u, u\right)=(T u, u)+\lambda(M u, u) \geqq(T u, u)$ for $\|u\| \geqq R$, independent of $\lambda$. If $T_{\lambda} u_{\lambda}=0$, it follows that either $\left\|u_{\lambda}\right\| \leqq R$ or $c\left(\left\|u_{\lambda}\right\|\right) \leqq 0$. Hence $\left\|u_{\lambda}\right\|$ is uniformly bounded. Hence $M\left(u_{\lambda}\right)$ is uniformly bounded. Choosing a sequence of $\lambda_{k} \rightarrow 0$, we see that $T\left(u_{\lambda}\right)=T_{\lambda}\left(u_{\lambda}\right)-\lambda M\left(u_{\lambda}\right)=-\lambda M\left(u_{\lambda}\right)$ $\rightarrow 0$. Since the $u_{\lambda}$ all lie in a fixed ball $B, 0$ lies in the closure of $T(B)$. By condition $(4)^{\prime}, 0$ lies in $T(B)$. q.e.d.

Proof of Theorem 2 Completed. Let $\lambda>0$ be fixed. We wish to show that 0 lies in $R\left(T_{\lambda}\right)$. We construct a one-parameter family of mappings $K_{t}$ of $V^{*}$ into $V^{*}$ for $0 \leqq t \leqq 1$, in the following way: For $t \in[0,1]$, let

$$
M_{t}(v, u)=(1-t)\{S(v, u)+C(u)+\lambda M(v)\}+t M(v) .
$$

For each fixed $t$ and $u, M_{t}(\cdot, u)$ is a continuous monotone mapping of $V^{*}$ into $V$, with $\left(M_{t}(v, u)-M_{t}\left(v_{1}, u\right), v-v_{1}\right) \geqq \lambda\left(M(v)-M\left(v_{1}\right)\right.$, $\left.v-v_{1}\right)$ and

$$
\begin{aligned}
\left(M_{t}(v, u), v\right) & \geqq\left(M_{t}(v, u)-M_{t}(0, u), v\right)+\left(M_{t}(0, u), v\right) \\
& \geqq \lambda(M(v), v)-g(\|u\|) v .
\end{aligned}
$$

Hence $\left(M_{t}(v, u), v\right) /\|v\| \rightarrow+\infty$ as $\|v\| \rightarrow+\infty$ (uniformly for bounded $u$ ) and by Theorem 5 of [4], there exists an unique element $v=K_{t}(u)$ for which

$$
M_{t}(v, u)=0 .
$$

Since the fixed points of the mapping $K_{0}$ are the zero points of $T_{\lambda}$, it suffices by the Leray-Schauder theorem [16] to verify the following:

(I) $K_{1}$ is a constant map. There exists a constant $M$ independent of $t$ such that if $K_{t}(u)=u$ for any $t$ in $[0,1]$, then $\|u\| \leqq M$.

(II) The map $(t, u) \rightarrow K_{t}(u)$ is a continuous map of $[0,1] \times V^{*}$ into $V^{*}$ such that for any bounded set $B$, the image of $[0,1] \times B$ is precom. pact.

Proof of (I). Since $M_{1}(v, u)=M(v), K_{1}$ is a constant map. If $K_{t}(u)=u$, we have $M_{t}(u, u)=0$. However,

$$
\left(M_{t}(u, u), u\right)=(1-t)(T u, u)+\{(1-t) \lambda+t\}(M u, u) \geqq \lambda(M(u), u)
$$

implies that in that case $(M(u), u) \leqq 0$, and $\|u\| \leqq M$ with a constant $M$ independent of $t$. q.e.d.

PROOF OF (II). It suffices to prove both of the following:

(a) If $u_{j} \rightarrow u, v_{j}=K_{t_{j}} u_{j} \rightarrow v, t_{j} \rightarrow t$, then $v=K_{t} u$.

(b) If $u_{j} \rightarrow u, t_{j} \rightarrow t, v_{j}=K_{t_{j}} u_{j} \rightarrow v$, then for a subsequence, $v_{j} \rightarrow v$. 
Proof of (a). We have $C\left(u_{j}\right) \rightarrow C(u)$, and for any $z$ in $V^{*}, S\left(z, u_{j}\right)$ $\rightarrow S(z, u)$. Since $M_{t_{j}}\left(v_{j}, u_{j}\right)=0$, if we set $R_{t}(v, u)=(1-t) S(v, u)$ $\{(1-t) \lambda+t\} M(v)$, we see that $R_{t_{j}}\left(v_{j}, u_{j}\right)=M_{t_{j}}\left(v_{j}, u_{j}\right)-\left(1-t_{j}\right) C\left(u_{j}\right)$ $\rightarrow(1-t) C(u)$. Since

$$
\left(R_{t_{j}}\left(v_{j}, u_{j}\right)-R_{t_{j}}\left(z, u_{j}\right), v_{j}-z\right) \geqq 0,
$$

passing to the limit as $j \rightarrow+\infty$, we obtain

$$
\left(-(1-t) C(u)-R_{t}(z, u), v-z\right) \geqq 0 .
$$

Using our previous remark with $Q(z)=R_{t}(z, u)$, we see that $R_{t}(v, u)$ $=-(1-t) C(u)$, i.e. $M_{t}(v, u)=0$ and $v=K_{t}(u)$. q.e.d.

Proof of (b). Since $C$ is compact and $\left\{u_{j}\right\}$ is bounded, we may assume by passing to a subsequence that $C\left(u_{j}\right) \rightarrow w_{1}$. Using the mapping $R_{t}$ defined in the proof of (a), we have

$$
R_{t_{j}}\left(v_{j}, u_{j}\right) \rightarrow-(1-t) w_{1} .
$$

Since

$$
\left(R_{t_{j}}\left(v, u_{j}\right)-R_{t_{j}}\left(v, u_{j}\right), v_{j}-v\right) \geqq \lambda\left(M(v)-M(v), v_{j}-v\right) \geqq 0
$$

and

$$
\left(R_{t_{j}}\left(v_{j}, u_{j}\right)-R_{t_{j}}\left(v, u_{j}\right), v_{j}-v\right) \rightarrow\left(-(1-t) w_{1}-R_{t}(v, u), 0\right)=0
$$

it follows that $\left(M\left(v_{j}\right)-M(v), v_{j}-v\right) \rightarrow 0$ and hence $v_{j} \rightarrow v$. q.e.d.

\section{BIBLIOGRAPHY}

1. F. E. Browder, Nonlinear elliptic boundary value problems, Bull. Amer. Math. Soc. 69 (1963), 862-874.

2. - Strongly nonlinear parabolic boundary value problems, Amer. J. Math. 86 (1964), 339-357. 301.

3. —-, Nonlinear elliptic problems. II, Bull. Amer. Math. Soc. 70 (1964) 299-

4. - Nonlinear elliptic boundary value problems. II, Trans. Amer. Math. Soc. 117 (1965), 530-550.

5. - Nonlinear equations of evolution, Ann. of Math. 80 (1964), 485-523.

6. - Multivalued monotone nonlinear mappings and duality mappings in Banach spaces, Trans. Amer. Math. Soc. 118 (1965), 338-351.

7. - Nonlinear initial value problems, Ann. of Math. 81 (1965), 51-87.

8. - Remarks on nonlinear functional equations, Proc. Nat. Acad. Sci. U.S.A. 51 (1964), 985-989.

9. - Existence and uniqueness theorems for solutions of nonlinear boundary problems, Proc. Sympos. Appl. Math. Vol. 17, Amer. Math. Soc., Providence, R. I., 1965, pp. 24-49.

10. - Nonlinear monotone operators and convex sets in Banach spaces, Bull. Amer. Math. Soc. 71 (1965), 780-785. 
11. - Nonlinear operators in Banach spaces and nonlinear boundary value problems, (to appear).

12. U. Dubinski, Some integral inequalities and solvability of degenerate quasilinear elliptic systems, Mat. Sb. 64 (1964), 458-480.

13. P. Hartman and G. Stampacchia, On some nonlinear elliptic differentialfunctional equations, (to appear).

14. M. A. Krasnoselski and Ya. B. Rutickii, Convex functions and Orlicz spaces, Moscow, 1958. (Transl. by Noordhoff, Groningen, 1961.)

15. J. Leray and J. L. Lions, Quelques résultats de Višik sur les problemes elliptiques nonlinéaire par le methode de Minty-Browder, Bull. Soc. Math. France 93 (1965), 97-107.

16. J. Leray and J. Schauder, Topologie et equations fonctionelles, Ann. Sci. Ecole Norm. Sup. (3) 51 (1934) 45-78.

17. G. J. Minty, On a "monotonicity" method for the solution of nonlinear equations in Banach spaces, Proc. Nat. Acad. Sci. U.S.A. 50 (1963), 1038-1041.

18. M. I. Višik, Quasi-linear strongly elliptic systems of differential equations in divergence form, Trudy Moscov. Mat. Obšc. 12 (1963), 125-184.

19. - Solvability of the first boundary value problem for quasilinear equations with rapidly increasing coefficients in Orlicz classes, Doki. Akad. Nauk. SSSR 151 (1963), 758-761.

University of Chicago 